\title{
Manejo químico de Rhynchospora cephalotes no Baixo Tocantins-PA
}

\author{
Mateus Gaia de Souza ${ }^{\mathrm{a}}{ }^{\oplus}$, Mariana Casari Parreira ${ }^{\mathrm{a}}{ }^{\odot}$, Jefferson dos Santos Martins ${ }^{\mathrm{a}}{ }^{\circledR}$, \\ Evaldo Morais da Silva ${ }^{\mathrm{a} *}$, Rafael Coelho Ribeiro ${ }^{\mathrm{a}}$ \\ a Universidade Federal do Pará, Brasil \\ *Autor correspondente (evaldomorais@ufpa.br)
}

\section{N F O}

\section{Keywords}

weed control

association of

herbicides

control

\begin{abstract}
A B S T R A C T
Chemical management of Rhynchospora cephalotes in Baixo Tocantins-PA.

Aggressive weeds, such as Rhynchospora cephalotes, are the main weeds in areas with perennial crops and degraded pastures in the Amazonian environment. Studies show that the association of phenoxyacetic acids with other classes of herbicides may be a viable alternative in the management of difficult to control plants. The objective of this study was to evaluate the post-emergence efficacy of 2,4-D herbicide, alone or in combination, in the control of Rhynchospora cephalotes. The experimental design was randomized blocks with 4 replications. Isolated doses of 2,4-D (502,5, 1,005 and 2,010 g e.a. ha $\left.{ }^{-1}\right)$ were applied as treatments and these were associated with picloran $\left(960 \mathrm{~g} \mathrm{e}^{\mathrm{a}}\right.$. ha $\left.\mathrm{h}^{-1}\right)$, triclopyr $\left(960 \mathrm{~g} \mathrm{e}\right.$.a. ha $\left.{ }^{-1}\right)$ and glyphosate $\left(1,110 \mathrm{~g}\right.$ e.a. $\left.\mathrm{ha}^{-1}\right)$, one more control without application. The best results at the end of the evaluations, 42 days after application, were obtained by the three treatments of the mixture between 2,4-D and glyphosate, which presented control averages of $R$. cephalotes, around $98 \%$. The association between the herbicides 2,4-D and glyphosate, at any dose used in this experiment, proved to be effective in controlling $R$. cephalotes, when applied in post-emergence.
\end{abstract}

\section{R E S U M O}

Plantas daninhas agressivas, como a espécie Rhynchospora cephalotes, são as principais infestantes de áreas com cultivos perenes e pastagens degradadas no meio amazônico. Estudos demonstram que a associação de ácidos fenoxiacéticos com outras classes de herbicidas podem ser uma alternativa viável no manejo de plantas de difícil controle. Objetivou-se com este trabalho avaliar a eficácia, em pós emergência do herbicida 2,4-D, isolado ou em associação, no controle de Rhynchospora cephalotes. O delineamento experimental utilizado foi em blocos ao acaso, com 4 repetições. Foram aplicados como tratamentos,

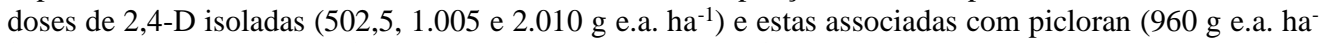
$\left.{ }^{1}\right)$, triclopyr (960 g e.a. ha $\left.{ }^{-1}\right)$ e glyphosate $\left(1.110\right.$ g e.a. ha $\left.{ }^{-1}\right)$, mais uma testemunha sem aplicação. Os melhores resultados no final das avaliações, 42 dias após a aplicação, foram obtidos pelos três tratamentos da associação 2,4-D e glyphosate, que apresentaram médias de controle de $R$. cephalotes, em torno de $98 \%$. A associação entre os herbicidas 2,4-D e glyphosate, em qualquer dose utilizada nesse experimento, se mostrou eficaz no controle da espécie $R$. cephalotes, quando aplicada em pós emergência. 


\section{INTRODUÇÃO}

A espécie Rhynchospora cephalotes (L.) pertencente à família Cyperaceae, popularmente chamada de capim-assapê ou rabo-de-fogete, distribui-se desde de o México à região da América do Sul, podendo comumente ser encontrada em grande parte das regiões brasileiras, especialmente no norte e nordeste do país (Schneider et al., 2017).

Por ser uma espécie extremamente agressiva, ela figura como uma das plantas daninhas mais importantes do meio amazônico, sobretudo no Baixo Tocantins (nordeste paraense), infestando principalmente áreas de cultivos perenes e pastagens degradadas. Seu manejo difícil, pois se reproduz através de rizomas que formam grandes touceiras, inviabilizando a prática da roçagem em detrimento do seu adensamento (Falcão-da-Silva et al., 2016). A crescente eficácia do controle químico de plantas daninhas em função do avanço biotecnológico na agricultura é cada vez mais acentuada, em comparação a outros métodos de controle utilizados na região amazônica, entretanto, a sustentabilidade dos sistemas deve ser prevalecida (Powles e Yu, 2010).

Diversos estudos apontam que a utilização de ácidos fenoxiacéticos associados a outras classes de herbicidas vem despontando como uma promissora alternativa no manejo de plantas daninhas de difícil controle (Takano et al., 2015). Além da combinação de herbicidas com diferentes mecanismos de ação, que contribui para que não ocorra a resistência de plantas daninhas a herbicidas (Bressanin et al., 2015).

Nesse contexto, objetivou-se com este trabalho avaliar a eficácia, em pós emergência do herbicida 2,4-D, isolado ou em associação, no controle de Rhynchospora cephalotes.

\section{MATERIAL E MÉTODOS}

O experimento foi realizado em condições de campo na Fazenda Monte Sião localizada no município de Oeiras do Pará-PA, região do Baixo Tocantins (latitude $02^{\circ} 19^{\prime} 24,2^{\prime} \mathrm{S}$ e longitude $49^{\circ} 45^{\prime} 51,1$ ' 'W), entre os meses de abril a junho de 2019. Onde a população de $R$. cephalotes utilizada no ensaio, encontrava-se em pleno período fisiológico vegetativo.

Foram coletadas amostras de solo da área de instalação do experimento antes da infestação de $R$. cephalotes para a determinação das características químicas e físicas do local, descritas na Tabela 1.

Foi utilizado o delineamento experimental de blocos ao acaso com quatro repetições, e as parcelas com dimensões de $3 \times 2 \mathrm{~m}\left(6 \mathrm{~m}^{2}\right)$, sendo 12 tratamentos herbicidas nas diferentes doses e associações, mais uma testemunha sem aplicação, descritos na Tabela 2.

Tabela 1 - Propriedades químicas e físicas do solo na camada $0-20 \mathrm{~cm}$, anterior a infestação de $R$. cephalotes. Oeiras do Pará-PA. 2019.

\begin{tabular}{ccccccccccccc}
\hline $\mathrm{pH}$ & M.O. & $\mathrm{P}$ & $\mathrm{K}$ & $\mathrm{Ca}$ & $\mathrm{Mg}$ & $\mathrm{SB}$ & $\mathrm{CTC}$ & $\mathrm{V}$ & & \multicolumn{3}{c}{ Granulometria (\%) } \\
\hline $\mathrm{CaC}_{12}$ & $\%$ & $\mathrm{mg} \mathrm{dm}^{3}$ & \multicolumn{3}{c}{$\mathrm{cmol} \mathrm{dm}^{3}$} & & $\%$ & Areia & Silte & Argila & $\begin{array}{c}\text { Classe/ } \\
\text { Textura }\end{array}$ \\
\hline 4,2 & 1,0 & 9,0 & 0,03 & 0,9 & 0,2 & 1,13 & 4,53 & 24,94 & 60,2 & 28,0 & 11,8 & $\begin{array}{c}\text { Argilo- } \\
\text { Arenoso }\end{array}$ \\
\hline
\end{tabular}

Fonte: Unithal (2019)

Tabela 2 - Lista de tratamentos utilizados aplicados em pós emergência para o controle de R. cephalotes. Oeiras do Pará (PA) 2019.

\begin{tabular}{|c|c|c|c|}
\hline Tratamentos & $\begin{array}{c}\text { Dose } \\
\text { g e.a. }{ }^{1} \text { ha }^{-1}\end{array}$ & $\begin{array}{c}\text { Dose } \\
\text { L p.c. }^{2} \text { ha }^{-1}\end{array}$ & Nome comercial \\
\hline 01. Testemunha & - & - & - \\
\hline 02. 2,4-D + Picloran & $502,5+960$ & $0,75+4,0$ & \\
\hline 03. 2,4-D + Picloran & $1.005+960$ & $1,5+4,0$ & DMA 806 BR + Padron \\
\hline 04. 2,4-D + Picloran & $2.010+960$ & $3,0+4,0$ & \\
\hline 05. 2,4-D + Triclopyr & $502,5+960$ & $0,75+2,0$ & DMA 806 BR + \\
\hline 06. 2,4-D + Triclopyr & $1.005+960$ & $1,5+2,0$ & Garlon $480 \mathrm{BR}$ \\
\hline 07. 2,4-D + Triclopyr & $2.010+960$ & $3,0+2,0$ & \\
\hline 08. 2,4-D + Glyphosate & $502,5+1.110$ & $0,75+3,0$ & \\
\hline 09. 2,4-D + Glyphosate & $1.005+1.110$ & $1,5+3,0$ & DMA 806 BR + Roundup Origi- \\
\hline 10. 2,4-D + Glyphosate & $2.010+1.110$ & $3,0+3,0$ & nal DI \\
\hline 11. 2,4-D & 502,5 & 0,75 & \\
\hline 12. 2,4-D & 1.005 & 1,5 & DMA 806 BR \\
\hline 13. 2,4-D & 2.010 & 3,0 & \\
\hline
\end{tabular}

${ }^{1}$ gramas de equivalente ácido por hectare; ${ }^{2}$ litros do produto comercial por hectare. 
Para a aplicação dos tratamentos foi utilizado um pulverizador costal, com pressão constante (mantida por $\mathrm{CO}^{2}$ comprimido) de $28 \mathrm{lbf} / \mathrm{pol} 2$, munido de barra com quatro bicos de jato plano ("leque") 110.02 , espaçados de $0,5 \mathrm{~m}$, com consumo de calda equivalente a $200 \mathrm{~L} \mathrm{ha}^{-1}$. As condições edáficas e climáticas no momento da aplicação foram: solo úmido, $21,8^{\circ} \mathrm{C}$ de temperatura do ar; $16,6^{\circ} \mathrm{C}$ de temperatura do solo; $67 \%$ de umidade relativa do ar. As condições climáticas durante o experimento estão apresentadas na Figura 1.

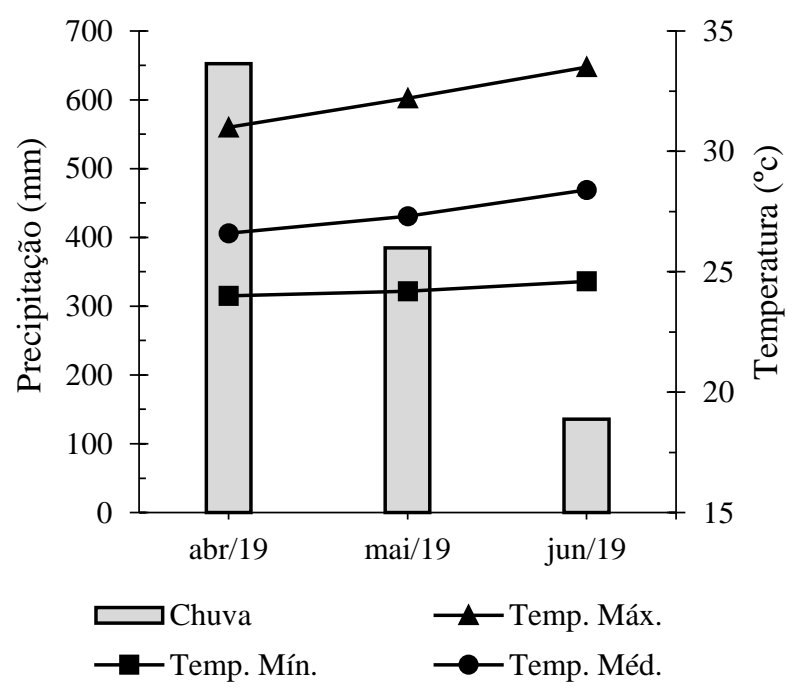

Figura 1 - Precipitação em milímetros e temperaturas (média, máxima e mínima) durante a realização do experimento. Oeiras do Pará-PA. 2019.

Aos 7, 14, 21, 28, 35 e 42 dias após a aplicação (DAA) dos herbicidas, foram realizadas avaliações visuais de controle segundo a escala de notas da EWRC (1964) atribuindo-se notas em porcentagem. Os efeitos das doses de 2,4-D e dos outros herbicidas utilizados quando significativos $(\mathrm{p}<0,01 \mathrm{e}$ $\mathrm{p}<0,05$ ), foram comparados pelo teste de Scott \& Knott (a 5\% de probabilidade) e o programa estatístico utilizado o Agroestat.

\section{RESULTADOS E DISCUSSÃO}

A tabela 3 apresenta o comportamento do capimassapê no decorrer do período experimental quando submetido aos tratamentos. Aos 7 Dias Após a Aplicação (DAA) a porcentagem de controle na maioria das tratamentos utilizados foi baixa, variando de $7,25 \%$ a $34,5 \%$, com os tratamentos 2,4-D na dose de 502,5 g e.a. ha ${ }^{-1}$,e 2,4-D na dose de 2.010 g e.a. ha ${ }^{-1}$,respectivamente (Tabela 3 ) devido a ação lenta dos herbicidas sistêmicos. Entretanto, ao associar o herbicida glyphosate, o controle foi maior, superando $58 \%$ atingindo até $75 \%$ controle, na maior dose associada, porém deve-se ressaltar que essa porcentagem de controle não é satisfatória de acordo com o Ministério de Agricultura e Pecuária (Mapa, 2019), no qual considera aceitável acima de $80 \%$ de controle.

Tabela 3 - Porcentagem de controle das plantas de R. cephalotes aos 7, 14, 21, 28, 35 e 42 dias após a aplicação (DAA), quando submetidas aos tratamentos testados, Oeiras do Pará (PA) 2019.

\begin{tabular}{|c|c|c|c|c|c|c|c|}
\hline \multirow{2}{*}{ Tratamentos } & \multirow{2}{*}{$\frac{\text { Dose }}{\text { g e.a. }{ }^{1} \text { ha }^{-1}}$} & \multicolumn{6}{|c|}{ Avaliação de controle (\%) } \\
\hline & & $7 \mathrm{DAA}^{2}$ & $14 \mathrm{DAA}^{2}$ & $21 \mathrm{DAA}^{2}$ & $28 \mathrm{DAA}^{2}$ & $35 \mathrm{DAA}^{2}$ & $42 \mathrm{DAA}^{2}$ \\
\hline 01. Testemunha & - & $0,00 \mathrm{~h}$ & $0,00 \mathrm{i}$ & $0,00 \mathrm{f}$ & $0,00 \mathrm{f}$ & $0,00 \mathrm{~g}$ & $0,00 \mathrm{f}$ \\
\hline 02. 2,4-D + Picloran & $502,5+960$ & $9,00 \mathrm{f}$ & $13,57 \mathrm{f}$ & $24,25 \mathrm{c}$ & $35,12 \mathrm{~d}$ & $54,37 \mathrm{c}$ & $74,55 \mathrm{~b}$ \\
\hline 03. 2,4-D + Picloran & $1.005+960$ & $17,47 \mathrm{e}$ & $15,65 \mathrm{f}$ & $23,87 \mathrm{c}$ & $50,02 \mathrm{~b}$ & $53,85 \mathrm{c}$ & $61,40 \mathrm{c}$ \\
\hline 04. 2,4-D + Picloran & $2.010+960$ & $18,9 \mathrm{f}$ & $32,50 \mathrm{~d}$ & $23,70 \mathrm{c}$ & $43,72 \mathrm{c}$ & $61,27 \mathrm{~b}$ & $62,95 \mathrm{c}$ \\
\hline 05. 2,4-D +Triclopyr & $502,5+960$ & $11,07 \mathrm{f}$ & $6,92 \mathrm{~h}$ & $20,65 \mathrm{c}$ & $36,40 \mathrm{~d}$ & $34,15 \mathrm{e}$ & $41,87 \mathrm{~d}$ \\
\hline 06. 2,4-D +Triclopyr & $1.005+960$ & $4,12 \mathrm{~g}$ & $7,10 \mathrm{~h}$ & $23,25 \mathrm{c}$ & $40,67 \mathrm{c}$ & $36,32 \mathrm{e}$ & $39,62 \mathrm{~d}$ \\
\hline 07. 2,4-D + Triclopyr & $2.010+960$ & $8,75 \mathrm{f}$ & $10,45 \mathrm{~g}$ & $17,75 \mathrm{~d}$ & $34,97 \mathrm{~d}$ & $43,75 \mathrm{~d}$ & $43,02 \mathrm{~d}$ \\
\hline 08. 2,4-D + Glypho. & $502,5+1.110$ & $59,16 \mathrm{c}$ & $76,27 \mathrm{c}$ & $92,37 \mathrm{c}$ & $97,97 \mathrm{a}$ & 98,37 a & $98,55 \mathrm{a}$ \\
\hline 09. 2,4-D+ Glypho. & $1.005+1.110$ & $68,40 \mathrm{~b}$ & $81,22 \mathrm{~b}$ & $87,02 \mathrm{~b}$ & $95,00 \mathrm{a}$ & 97,47 a & 98,27 a \\
\hline 10. 2,4-D + Glypho. & $2.010+1.110$ & $75,98 \mathrm{a}$ & $92,87 \mathrm{a}$ & 94,37 a & $97,47 \mathrm{a}$ & $97,65 \mathrm{a}$ & $98,15 \mathrm{a}$ \\
\hline 11. 2,4-D & 502,5 & $7,25 \mathrm{f}$ & $10,87 \mathrm{~g}$ & $7,47 \mathrm{e}$ & $26,75 \mathrm{e}$ & $285 \mathrm{f}$ & $32,12 \mathrm{e}$ \\
\hline 12. $2,4-\mathrm{D}$ & 1.005 & $9,52 \mathrm{f}$ & $8,15 \mathrm{~h}$ & $15,57 \mathrm{~d}$ & $24,87 \mathrm{e}$ & $30,37 \mathrm{f}$ & $34,62 \mathrm{e}$ \\
\hline 13. 2,4-D & 2.010 & $34,57 \mathrm{~d}$ & $24,20 \mathrm{e}$ & $24,15 \mathrm{c}$ & $28,25 \mathrm{e}$ & $35,95 \mathrm{e}$ & $42,50 \mathrm{~d}$ \\
\hline F Tratamento & & $594,37 * *$ & $711,25 * *$ & $530,75 * *$ & $524,04 * *$ & $1227,88 * *$ & $915,47 * *$ \\
\hline $\mathrm{DMS}^{3}$ & & 8,52 & 8,2 & 8,17 & 5,72 & 3,34 & 3,53 \\
\hline $\mathrm{CV}(\%)^{4}$ & & $0,00 \mathrm{~h}$ & $0,00 \mathrm{i}$ & $0,00 \mathrm{f}$ & $0,00 \mathrm{f}$ & $0,00 \mathrm{~g}$ & $0,00 \mathrm{f}$ \\
\hline
\end{tabular}

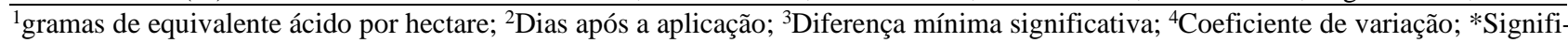
cativo ao nível de $5 \%$ de probabilidade. Médias seguidas de mesma letra, na coluna, não diferem pelo teste de Scot t\& Knott a 5\% de significância. 
Aos 14 DAA nota-se, na Tabela 3, que as maiores doses do 2,4-D, 1.005 e $2.010 \mathrm{~g}$ e.a. ha ${ }^{-1}$, associadas com glyphosate a 1.110 g e.a. ha ${ }^{-1}$ foram eficazes no controle de $R$. cephalotes, com médias em torno de 81,22 e $92,87 \%$, respectivamente. Resultados semelhantes foram presenciados por Takano et al. (2015) no qual associaram os herbicidas 2,4D e glyphosate, sendo essas associações os melhores tratamentos no controle de plantas daninhas consideradas de difícil manejo em áreas agrícolas. Em contrapartida, os tratamentos à base de 2,4-D + triclopyr e 2,4-D, isolado nas maiores doses, foram observados os menores níveis de controle para o mesmo período (Tabela 3).

Nesse contexto, Dan et al. (2009) descreve que herbicidas com mecanismo de ação sistêmico, que apresentam translocação na planta, como no caso do 2,4-D e dos demais utilizados no presente ensaio, apesar de sofrerem incremento nas dosagens, a sua ação inicial lenta, proporcionando resultados percentuais de ineficiência nos primeiros estádios das avaliações de controle. Esses mesmos autores constataram ainda que somente aos 28 DAA da maior dose testada de 2,4-D (1.340 g e.a. ha-1) foi possível observar eficácia no controle percentual, em torno de $84 \%$.

Verifica-se também, pela análise da Tabela 3, que a partir dos 21 DAA os tratamentos com o herbicida 2,4-D em associação com glyphosate evoluíram significativamente o nível de controle, superando $94 \%$, na maior dose utilizada, sendo considerado, nesta época de avaliação a associação mais eficiente no controle de $R$. cephalotes, sendo observado este comportamento ao restante do período experimental. Ramos e Durigan (1996), relatam haver sinergismo entre o 2,4-D e glyphosate, apresentando melhores resultados para o controle de gramíneas do que se fosse aplicado em forma isolada.

Ainda nos 21 DAA, os tratamentos com as duas maiores doses isoladas de 2,4-D proporcionaram as menores médias de controle, juntamente com o tratamento entre 2,4-D (2.010 g e.a. ha $\left.{ }^{-1}\right)$ e triclopyr a $960 \mathrm{~g}$ e.a. ha ${ }^{-1}$. Novamente os pesquisadores Dan et al. (2009) ao utilizar o herbicida 2,4-D na dose de $1.005 \mathrm{~g}$ e.a. ha ${ }^{-1}$ observaram que o tratamento não foi eficiente no controle da gramínea avaliada, sendo necessário a associação com outro herbicida. A Figura 2 representa a porcentagem de controle proporcionada pelos herbicidas aplicadas de forma associada ou isolada, ao se fazer a média de controle das três doses utilizadas, no decorrer do período experimental. É possível observar que o percentual de controle dos tratamentos com associação entre 2,4-D e glyphosate, desde a primeira avalia- ção (7 DAA), apresentaram controle maior aos demais tratamentos, sendo observado também que a partir dos 28 DAA, o controle tornou-se estável até o final das avaliações (42 DAA), com valores acima dos $98 \%$. Valores eficazes de controle similares a esses foram encontrados por Galli (1991), no qual constatou que aos 28 DAA, a associação pronta de 2,4-D + glyphosate $\left(2.240\right.$ g e.a. ha $\left.{ }^{-1}\right)$, controlou $98 \%$ de plantas daninhas em área de cultivo comercial.

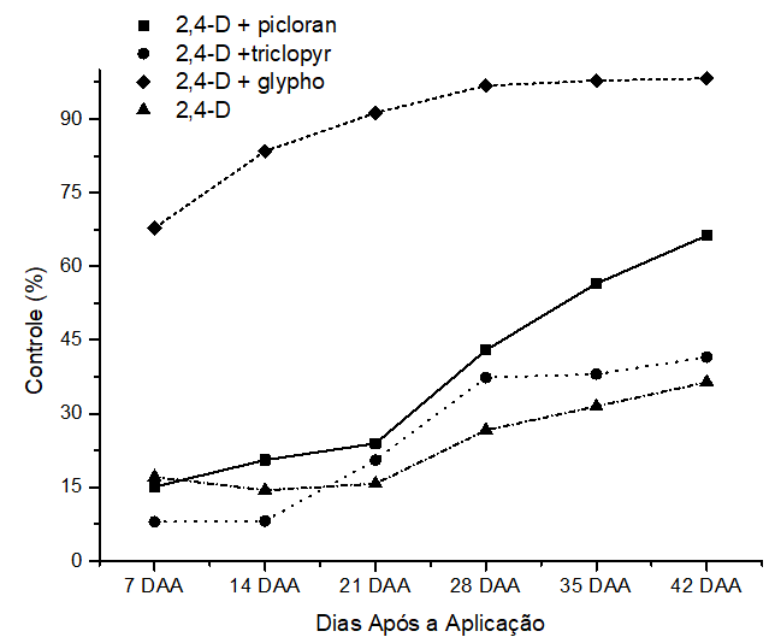

Figura 2 - Controle (\%) de R. cephalotes pelas médias das doses utilizadas dos tratamentos aplicados de forma associada ou isolada, no decorrer do período experimental. Oeiras do Pará-PA. 2019.

Como observado na Tabela 3 e Figura 2, apesar dos tratamentos à base da associação entre 2,4-D e picloran obterem baixos controles em todas as avaliações realizadas, aos 28 DAA, foram os que apresentaram maior evolução percentual no controle da $R$. cephalotes, sendo superados apenas pelos tratamentos da associação entre 2,4-D e glyphosate, no final do período experimental. No entanto, os pesquisadores Oliveira et al. (2009) ao testar diferentes herbicidas no controle de plantas daninhas de difícil manejo, verificou que a dose mínima da associação 2,4-D + picloram $\left(480+130\right.$ g e.a. ha $\left.{ }^{-1}\right)$, inferior a mínima aplicada no presente trabalho, proporcionou controle aceitável durante as avaliações visuais. Do mesmo modo, Caldeira et al. (2014) descrevem que a utilização desses produtos de acordo com as respectivas doses recomendadas pelo fabricante, quando em associação, propiciaram controle final acima de $99 \%$ das plantas daninhas testadas.

Com relação aos tratamentos com aplicações de doses isoladas de 2,4-D observou-se que, assim como os demais tratamentos, aos 28 DAA houve um acréscimo de controle, no entanto, juntamente 
com a associação de 2,4-D + triclopyr, foram os que demonstraram os menores percentuais de controle de $R$. cephalotes entre todos os avaliados (Figura 2, Tabela 3).

O herbicida 2,4-D na dose de 1.340 g e.a. ha ${ }^{-1}$, também não mostrou efeito significativo no controle de plantas infestantes de áreas de pastagens em trabalho desenvolvido por Krenchinski et al. (2015), contudo, o herbicida triclopyr (240 g e.a. $\mathrm{ha}^{-1}$ ) em associação com outro ácido piridiniloxialcanoico apresentou médias de $100 \%$ de controle desde os 14 primeiros dias após a aplicação.

Aos 35 e 42 DAA os tratamentos obtiveram comporatamentos semelhantes, não demonstarando grandes variações de controle entre as avaliações, sendo a associação de 2,4-D e glyphosate, em qualquer dose utilizada, a que proporcionou maior porcentagem de controle, alcançando 98,35\% (35 DAA) e $98,55 \%$ (42 DAA) como observado na Tabela 3. Os menores controles foram propiciados novamente pelo herbicida 2,4-D, aplicado de forma isolada (Figura 2).

\section{CONCLUSÕES}

A associação entre os herbicidas 2,4-D e glyphosate, em qualquer dose utilizada nesse experimento, se mostrou eficaz no controle da espécie $R$. cephalotes, quando aplicada em pós emergência.

\section{REFERÊNCIAS BIBLIOGRÁFICAS}

Bressanin FN, Giancotti PRF, Neto NJ, Amaral CL, Alves LCA. Eficácia de herbicidas aplicados isolados em pré e pós-emergência no controle de mucuna-preta. Agrária, v.10, n.3, p.426-431, 2015.

https://doi.org/10.5039/agraria.v10i3a5337

Caldeira DSA, Do Amaral VN, Casadei RA, De Barros LV, Netto Figueiredo Z. Controle de plantas daninhas em pastagem usando doses e misturas de herbicidas. Enciclopédia Biosfera. v.10, n.18, p.1052-1060, 2014.

Dan HÁ, Barroso ALL, Procópio SO, Moraes Dan LG, Oliveira Neto AM, Guerra N, Braz GBP. Controle Químico de plantas voluntárias de soja Roundup Ready. Revista Brasileira de Herbicidas, v.8, n.3, p.96-101, 2009. https://doi.org/10.7824/rbh.v8i3.72

European Weed Research Council - EWRC. Report of the 3nd and 4th meetings of EWRC. Committee of Methods in Weed Research. Weed Research, v.4, n.1, p.88, 1964. https://doi.org/10.1111/j.1365-3180.1964.tb00271.x

Falcão-da-Silva M, Souza Filho APS, Gurgel ESC, Bastos MNC, Dos Santos JUM. Plantas Daninhas na Amazônia. Belém: Museu Paraense Emílio Goeldi, 188p. 2016.

Galli AJB. Avaliação da eficiência de glyphosate em mistura com diversos produtos, no controle de Commelina virginica (trapoeraba) em citros. In: Congresso Brasileiro de
Herbicidas e Plantas Daninhas, 1991, Brasília. Resumos, Brasília: SBHED, 1991, p.104-105.

Krenchinski FH, Albrecht AJP, Albrecht LP, Cesco VJS, Rodrigues DM, Victória Filho R. Application rates and herbicide in weed control in pasture. Revista Brasileira de Herbicidas, v.14, n.4, p.271-279, 2015. https://doi.org/10.7824/rbh.v14i4.435

MAPA - Ministério da agricultura Pecuária e Abastecimento. SISLEGIS: Sistema de Consulta à Legislação. Disponível em: <http://sistemasweb.agricultura.gov.br/sislegis/ action/detalhaAto.do?method=consultarLegislacaoFederal $>$. Acesso em: 26 jul. 2019.

Oliveira AR, Freitas SP, Vieira HD. Controle de Commelina benghalensis, $C$. erecta e Tripogandra diuretica na cultura do café. Planta Daninha, v.27, n.4, p.823-830, 2009. https://doi.org/10.1590/S0100-83582009000400021

Powles SB, Yu Q. Evolution in Action: Plants Resistant to Herbicides. Annual Review of Plant Biology, v.61, n.1, p.317-347, 2010. https://doi.org/10.1146/annurev-arplant-042809-112119

Ramos HH, Durigan JC. Avaliação da eficiência da mistura pronta de glyphosate $+2,4-\mathrm{D}$ no controle da Commelina Virginica L. em citros. Planta Daninha, v.14, n.1, p.33-41, 1996.

https://doi.org/10.1590/S0100-83581996000100004

SBCPD - Sociedade Brasileira da Ciência das Plantas Daninhas. Procedimentos para instalação, avaliação e análise de experimentos com herbicidas. Londrina: 1995. 42p.

Schneider LJC, Bastos MDNDC, Costa Neto SVD, Gil ADSB. Synopsis of the genus Rhynchospora (Cyperaceae) in the sandy coast of Pará state, Brazil. Rodriguésia, v.68, n.2, p.653-670, 2017. https://doi.org/10.1590/2175-7860201768222

Takano HK, Oliveira Jr RS, Constantin J, Biffe DF, Franchini LHM, Braz GBP. et al. Efeito da adição do 2,4$\mathrm{D}$ ao glyphosate para o controle de espécies de plantas daninhas de difícil controle. Revista Brasileira de Herbicidas, v. 2, n. 1, p. $1-13,2013$

https://doi.org/10.7824/rbh.v12i1.207 